$\$=-1$

\title{
Detection of Driver Drowsiness using Eye Blink Sensor
}

\author{
Kusuma Kumari B.M ${ }^{1}$, Sampada Sethi ${ }^{2}$, Ramakanth Kumar $\mathbf{P}^{3}$, Nishant Kumar ${ }^{4}$, \\ Atulit Shankar ${ }^{5}$ \\ ${ }^{1}$ Assistant Professor, Department of Computer Science, University College of Science, \\ Tumkur University, Tumakuru, Karnataka \\ ${ }^{2,4,5}$ Student, ${ }^{3}$ Professor, Department of Information Science and Engineering, \\ R.V. College of Engineering, Bangalore, Karnataka \\ *Corresponding Author E-mail: ${ }^{1}$ kusuma.kuku@gmail.com, ${ }^{2}$ sam.sethi1395@gmail.com, \\ ${ }^{3}$ ramakanthkp@rve.edu.in, ${ }^{4}$ n1994kumar@gmail.com \\ 5atulit.shankar@yahoo.com
}

\begin{abstract}
Accidents due to driver drowsiness can be prevented using eye blink sensors. The driver is supposed to wear the eye blink sensor frame throughout the course of driving and blink has to be for a couple of seconds to detect drowsiness. Any random changes in steering movement leads to reduction in wheel speed. The threshold of the vibration sensor can be varied and accordingly action can be taken. The outcome is that the vibrator attached to eye blink sensor's frame vibrates if the driver falls asleep and also the LCD displays the warning messages. The wheel is slowed or stopped depending on the condition. This is accompanied by the owner being notified through the GSM module, so the owner can retrieve the driver's location, photograph and police station list near to driver's location. This is how the driver can be alerted during drowsiness and the owner can be notified simultaneously
\end{abstract}

Keywords: eye blink sensor, LCD, Microcontroller, Drowsy Driver Detection System

\section{Introduction}

For any vehicle accidents driver's faults are the most accountable aspect to cause dangerous problem to the society. Many drivers cannot control the vehicles due to different reasons it may cause severe accidents and sometime death. For vehicle accidents various factors involved such as drunk driving, over speeding, many distractions like texting while driving, talking with others, playing with children etc. one of the important factor is sleeping on the wheel. People know the dangerous of alcohol consumption and run the vehicles but they not understand the seriousness of fatigue driving. In India, Ministry of Road Transport and Highway released a report in 2015, every day around 1,374 accidents may happen and almost 400 people deaths occur. Every hour because of vehicle accidents approximately 57 road accidents and 17 people dies. In that 54.1 percent of people are in the age group of 15 to 34 years are killed in vehicle accident. The Government of India, Ministry of Road Transport and Highway Government of India prepare a strategy to diminish the amount of motorway accidents and losses by $50 \%$ by 2020 .

Globally vehicle accidents have seemed one of the major community health problems. In India almost 5 lakh road accidents happened in the year 2015. A fatigue Driver those who falls asleep at the move fails to control the vehicle, not possible to take immediate action and results in a crash so it is necessary to monitor the drowsiness of the driver to prevent accidents.

\section{Literature Survey}

Automatic driver drowsiness can be detected using artificial intelligence and visual information.

System is to detect, track and examine face and eyes of drivers for this different real vehicle image of drivers are taken to validate the algorithms.

It is a real time system work in different light conditions [1]. The numbers of accidents are increased due to several factor, one of the main factor is that driver fatigue. Driver's sleepiness is also implemented using video based approach. This system is noninvasive and human related elements are used. Band power and Empirical Mode Decomposition methods are used to investigate and extract the signal, SVM (Support Vector Machine) used to confirm the analysis and to categorize the state of vigilance of the driver [2].

The system designs to find the drivers drowsiness using the hypothesis of Bayesian networks. The interaction between driver and vehicle features are extracted to get reliable symptoms of driver drowsiness. It presents more suitable and accurate strategies to design drowsy driver detection system [3].

Brain and visual activity is used in drowsiness detection system. Electroencephalographic (EEG) channel used to monitor the brain activity. Diagnostic techniques and fuzzy logic are used in EEGbased drowsiness detector. Using blinking detection and characterization for visual activity monitored. Electrooculographic (EOG) channel are used to extract the Blinking features [4].

Image processing and pattern classification used to take the driver facial pictures, tracking the features of driver face and categorizing the driver's sleepiness level. 17 different features 
points are determined after examining the facial muscle activities using Active Appearance Model (AAM). K-Nearest-Neighbor method applied to categorize sleepiness into 6 levels, driver's smile also detected with this method [5].

Head posture estimation method is used for detection of drowsy driver. In this method Viola and Jones algorithm for driver face detection [6].

This method is nonintrusive and sturdy for finding the driver drowsiness in real time. Support Vector Machine (SVM) is using for extracting the face from video frames and Circular Hough Transform (CHT) is useful for mouth and eye state analysis [7]. In this approach machine learning used to determine the human behavior during driver drowsiness, for this 30 different facial actions including eye blink, yawning and head movements are collected to detect the driver drowsiness [8].

\section{Methodology}

The concept of drowsy driver detection system focuses on the functioning of all sensor modules used in the project. This helps explain the inputs received by modules and the outputs they produce.

\subsection{Eye Blink Sensor}

This sensor module consists of the eye blink sensor frame, the IR sensor and a relay. The vibrator device is connected to the eye blink sensor frame which is to be worn by the driver. This vibrator vibrates whenever an accident occurs or the driver falls asleep. The frame consists of the IR transmitter which transmits the IR rays towards the driver's eyes and an IR receiver which receives the reflected rays when the eyes are closed. The relay provides the extra current required by this module and hence is also connected to the SST microcontroller board [9].

\subsection{LCD}

LCD is a device which displays the messages in case the driver falls asleep or accident occurs. The messages displayed are "SLEEPING" and "ACCIDENT OCCURRED" as per the situation. All the modules are connected to it so that the particular signals can be received and hence message could be displayed. It uses the power supply from the SST microcontroller board displays a "WELCOME" message. It also provides $5 \mathrm{~V}$ power to other modules [10].

\subsection{LM317PSU Board}

This board is connected to the relay chip which in further connected to the eye blink sensor system module. This board's main purpose is to fulfill the power requirements of the eye blink sensor module.

\subsection{Motor}

The motor acts as the wheel of the vehicle and it rotates when the power is supplied to it through L298 chip. The speed of rotation is slowed down when the driver falls asleep as detected by the eye blink sensor, in the other case the wheel is stopped when the accident occurs.

\subsection{Vibration Sensor}

The purpose of this sensor is to sense any jerk given to the vehicle which is the emulation of the accident occurrence in real time This receives power from the $5 \mathrm{~V}$ port of the LCD.

The output produces and sends signals to dc motor driver and stops the rotation of the wheel, that is, the motor.

\subsection{L298 Motor Driver}

The DC motor receives power from this motor driver, which in turn is powered from the SST microcontroller board. The enable pins of this driver should be high to send the power to the motor.

\subsection{GSM Module}

The GSM module used is GSM-SIM300. This module's primary function is to send an audio warning message to the owner's registered number that is, in case of any accident the owner is notified by it android application which plays an audio saying "accident occurred, kindly check your phone". A sim card is to be fixed in this module to simultaneously send a text message on the owner's phone.

\subsection{Accelerometer 3-Axis and ADC0809}

The accelerometer used is ADXL335 3-axis which acts as steering and measures any increase in the speed. The reference speed taken in this project is $150 \mathrm{kmph}$. As the vehicle turns randomly, the accelerometer generates analog values which are further given to the ADC0809, the converter used in this project. These digital values in turn act as inputs to the dc motor, the LCD and the vibrator.

\subsection{SST Microcontroller}

This is the main working unit which connects all the inputs and outputs to its 4 ports namely, P0 P1 P2 P3 and each of them have 8-pins which act as pins for input and output. The main ac dc power supply $\mathrm{f} 12 \mathrm{~V}$ is given to this board. It has a huge RAM and ROM which helps the program run faster as compared to other microcontrollers and also provides extra memory storage capacity [11].

\section{Implementation}

\subsection{Hardware Requirements}

The various sensors form the hardware part of the project and power supply is AC DC $12 \mathrm{~V}$ adapter. Other hardware modules are specified as follows:
1) PIC16F877A.
2) CAN Transceivers
3) Alarm circuit.
4) SST Micro Controller
5) Power Supply
6) IR eye blink sensor
7) LM 358 comparator
8) Accelerometer
9) LCD
10) Vibrator Output devices

\subsection{Software Requirements}

For the implementation of the modules, the coding language is specified in this part and also the platforms used for coding and the android application development:
1) Keil $\mathrm{C}$ compiler
2) Embedded $\mathrm{C}$
3) Android studio

\subsection{General Constraints}

To developing the system the subsequent constraints were kept in mind:

- $\quad$ The IR eye blink sensor's transmitter and receiver should be in a straight line. 
- There shall be no element of doubt regarding the correctness of the distance measured.

- The system must have the android app for location detection since phone's processor is faster than that used by GSM module.

- $\quad$ The system should be robust.

\subsection{Development Methods}

The development methods decide a large section of how the final system functions, and thus care is taken to ensure that the best practices, tools and equipments are used. The system will be developed using Keil IDE. The programming language embedded $\mathrm{C}$ will also be used. Certain sections of the system will be ported android application development for performance enhancement. The main modules would be microcontroller, IR sensor, LM358 comparator, accelerometer, LCD and android phone [12].
Drowsy driver detection system is divided into subsystems and these sub systems gives some interconnected services. Architecture design can be defined as proving a framework for sub-system control, in the first stage of the design process all subsystems of the drowsy driver detection system are identified and hence, the design process output is a output of this a explanation of the software architecture. A basic structural framework for a system can be established through architectural design process. The identification of the major components of the system and communications between these components is considered under this.

The system architecture below portrays the blocks required for the implemented system. Figure 1 outlines the system architecture. The sensors need to detect respective parameters and pass the signals to microcontroller. The outputs from SST are used as inputs by various output devices to control the speed and display the message [13].

\subsection{System Architecture}

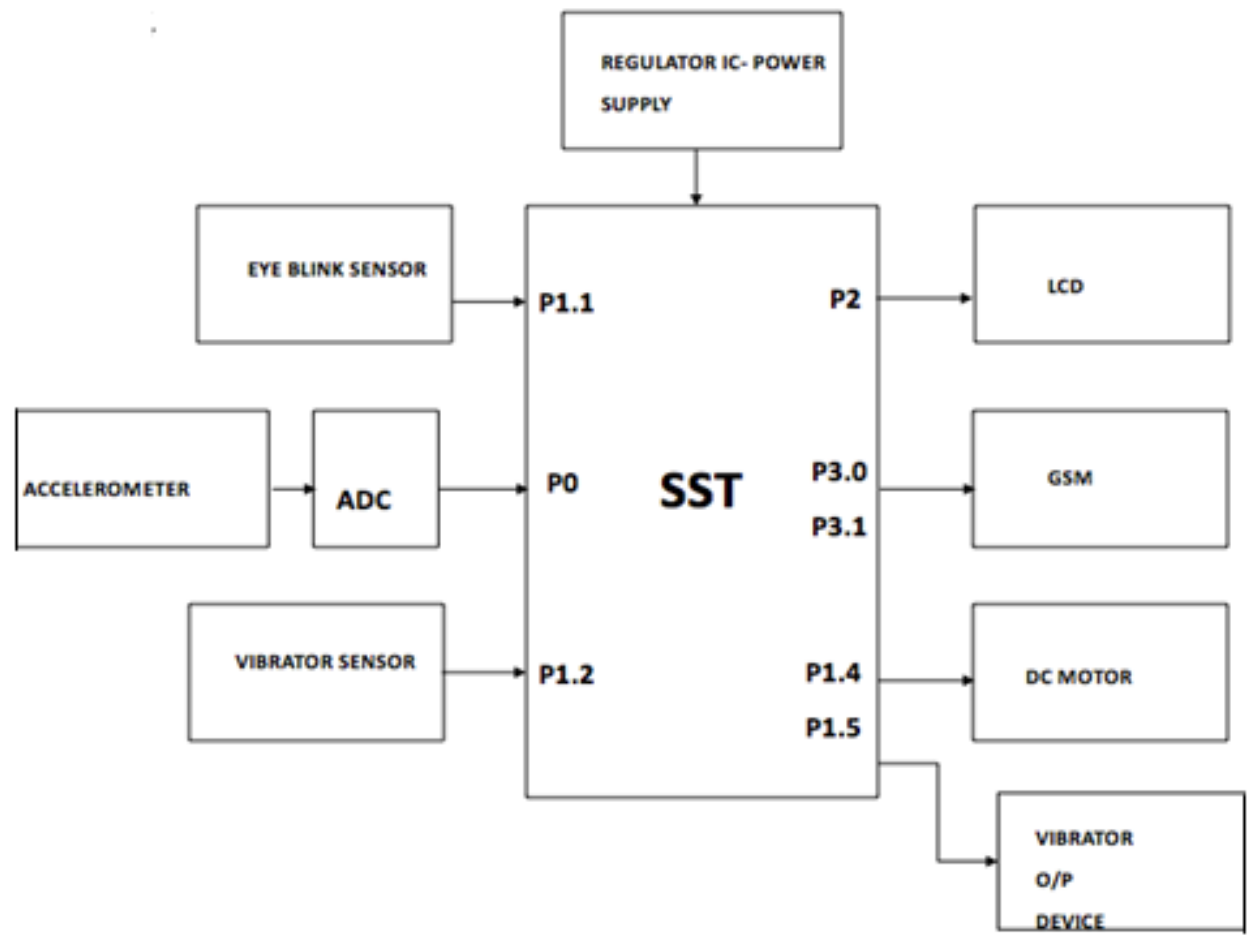

Fig. 1: System Architecture

Figure 2., depicts the structure chart of the complete system. The structure chart contains all the components of the system. 


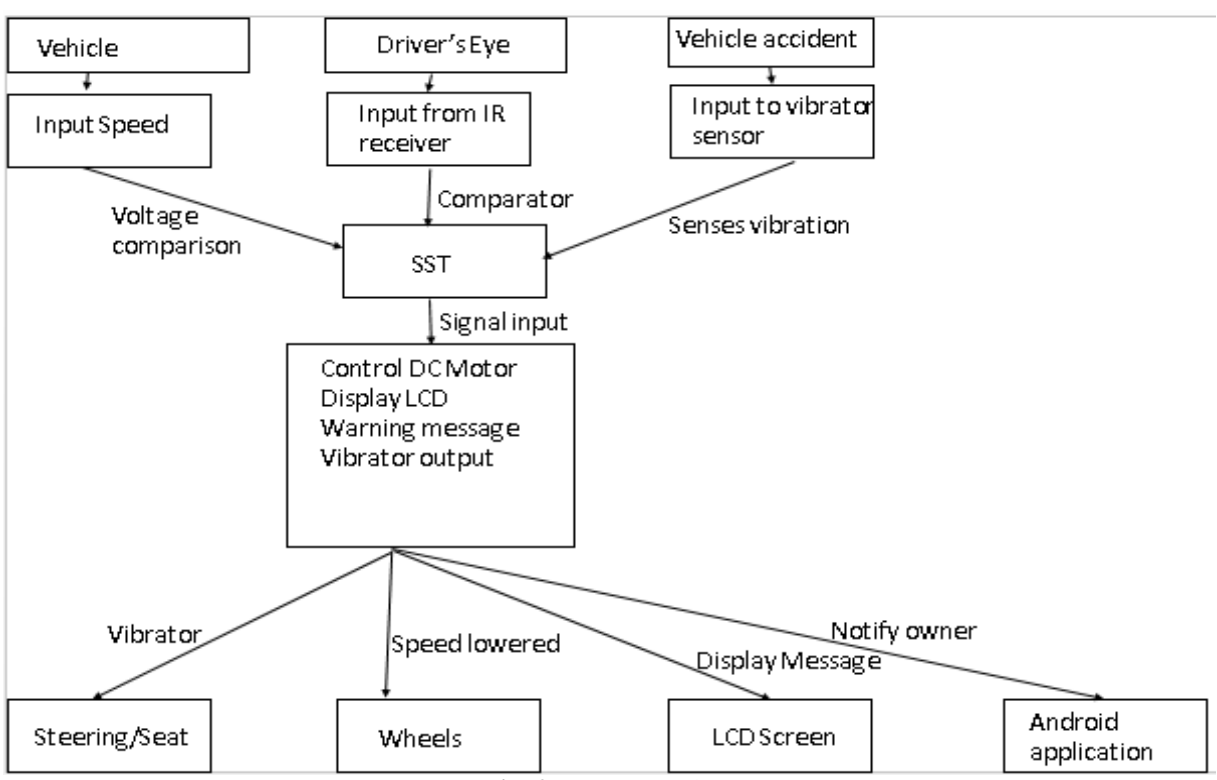

Fig. 2: Structure Chart

\subsection{Input Module}

This section is responsible for taking in crisp values of the vehicle, eye blink movements any vibration caused to vehicle due to accident.

\section{- $\quad$ Purpose}

The purpose of this module is obtaining values from each sensor and comparing them with reference values.

\section{- $\quad$ Functionality}

The functionality of this module is to obtain the raw values and pass signals to SST.

\section{- Input}

The input to this module are the crisp values of vehicle speed, IR from eye blink sensor and values from shock due to accident.

\section{- Output}

The output of this module is fed into SST80C51 microcontroller.

\subsection{SST Microcontroller}

This section contains three inputs and outputs to various output devices. It receives the signals from the previous modules

\section{- $\quad$ Purpose}

The purpose of this module is to pass the received values as input to output devices.

\section{- $\quad$ Functionality}

This module provides inputs to the output devices.

- Input

The inputs would be outputs received by input devices.

\section{- Output}

The output of SST would be passed to other output devices so various functionalities could be carried out as demanded by project.

\subsection{Output Devices}

This section contains DC motor, LCD, vibrator, GSM module which perform their respective functionalities.

\section{- $\quad$ Purpose}

The purpose of this module is to carry out respective functionalities such as slowing down the vehicle speed, alerting the driver, notifying the owner and displaying message on the LCD.

\section{- $\quad$ Functionality}

This module takes the input from the SST microcontroller through ports and carries out intended goals.

\section{- Input}

The inputs to this module are signals from ports of microcontroller.

\section{Output}

The output of SST would be passed to other output devices so various functionalities could be carried out as demanded by project.

\subsection{Aid for All 8051 Alternatives}

One of the most fastest growing Microcontroller Architecture are 8051 alternatives, today nearly more than 400 device alternatives available with various silicon vendors. For large applications Philips 80C51MX architecture the New extended 8051 devices are used because of the large storage capacity with several Mbytes. Keil also provides different Development tools for best support for these different variants are recorded below [14].

\subsection{Bit 80C51 5 V Low Power 64 Kb Microcontroller}

The P89V51RD2 is an 80C51 microcontroller with $64 \mathrm{kB}$ Flash and $1 \mathrm{kB}$ of data RAM. These devices are designed to be drop-in and software-compatible replacements for the popular P89C51RB2/RC2/RD2 devices. It supports both the In-System Programming (ISP) and In-Application Programming (IAP) boot codes with 12-clock (default) or 6-clock mode selection.it contains three 16 bit times/counters. In-System programming and InApplication Programming on chip flash user code memory is 64 $\mathrm{kb}$. Operating frequency in $12 \mathrm{x}$ mode is $0 \mathrm{MHz}$ to $40 \mathrm{M} \mathrm{Hz}$. Nonvolatile data storage is the 128 -B page erase for efficient use of code memory [16].

\subsection{PCA}

The Programmable Counter Array (PCA0) gives enhanced timer functionality and requiring less CPU involvement compare to the standard 8051 counter/timers. Dedicated 16-bit counter/timer and five 16-bit capture/compare modules are there in PCA. Each capture/compare module has its own associated I/O line (CEXn) which is routed through the Crossbar to Port I/O when enabled [17]. They can be programmed in any of the following modes: Rising or falling edge timer, Software timer, High speed output and PWM 


\subsection{Accelerometer}

An accelerometer is an electromechanical device. Acceleration force will measure using this device, due to cause of gravity i.e. $g$ force it shows acceleration and it measures in $g$ units. It is used in tilt-sensing applications as well as dynamic acceleration resulting from motion, shock, or vibration.

The ADXL335 is a popular three-axis analog accelerometer IC, which reads off the $\mathrm{X}, \mathrm{Y}$ and $\mathrm{Z}$ acceleration as analog voltages. Complete 3-axis acceleration measurement gives the ADXL335, within range $\pm 3 \mathrm{~g}$ in the $\mathrm{x}, \mathrm{y}$ and $\mathrm{z}$ axis. Analog voltages are proportional to the acceleration are the output signals of this module. Poly silicon surface-micro machined sensor and signal conditioning circuitry contains in ADXL335 [18].

\subsection{Eye Blink Sensor}

To observe the solidness of the traffic, we will be placing a few sets of IR transmitter \& receiver sensors on the side of the roads. On side IR transmitter device will be installed \& right opposite to the IR transmitter, an IR receiver will be positioned. This set of IR transmitter \& receiver will be placed at interim distance on the road [19].

The IR transmitters are attached to supply, so that they will transmit high signal all the time. The IR receivers are attached to the comparator circuit, to get digital signals. A low power operational amplifier LM324 IC has been used to build a comparator circuit. Two set of LM324 IC has been used in this project [20].

\subsection{Vibration Sensor}

The vibration sensor buffers a piezoelectric transducer. The transducer generates the voltages, when the transducer moved from neutral axis, distortion makes strain within the piezoelectric element. Security purpose only this vibration sensor decorator is designed and it produced alarm through movement or vibration, and it sends a signal to control panel, a new type of multi directional security vibration detector with multi directional detection also developed [21].

\section{Testing}

For unit testing the following contents are suggestive testing strategy in the system [22]:

- The design description characteristics and source code for each module to be trialed are first reviewed.

- An evaluation committee is responsible for validation of the Unit Test Plan.

- A trial "stub" is created to allocate input to or fetch output from the trial module.

- The code is then compiled in the trial environment to check for any anomalies.

- The trials are executed and data received out of the verified software are compared with the expected, as recorded in the Unit Test Plan.

- The code is retested and results are recorded, when an updated version is available

- Final report form archived from that unit is said to have cleared all trials.

- $\quad$ Every possible tweaks/ related to software requirements and draft documents are provided.

\section{Results}

\subsection{The Drowsy Driver Detection System}

The results obtained by running the module are as follows:
- The owner's phone receives an audio warning message.

- The owner can retrieve the location of the driver by choosing the "location" option.

- The owner can also get a list of driver's nearby police stations. With this, the photograph of the driver can be sent to owner's email address as specified in the clamped phone.

- The wheel/motor is stopped as soon as an accident occurs, the vibrator in the eye blink sensor frame vibrates and displays a message on the LCD.

- When the driver falls asleep, the vibrator vibrates and the LCD displays the message. Along with this, the vehicle speed is automatically reduced.

- When the accelerometer is tilted randomly, that acts as the steering, a message is displayed on the LCD the speed of the vehicle is reduced.

- With all of the above mentioned, the android applications send and receives details simultaneously.

\subsection{Vibration Sensor Senses Vibration}

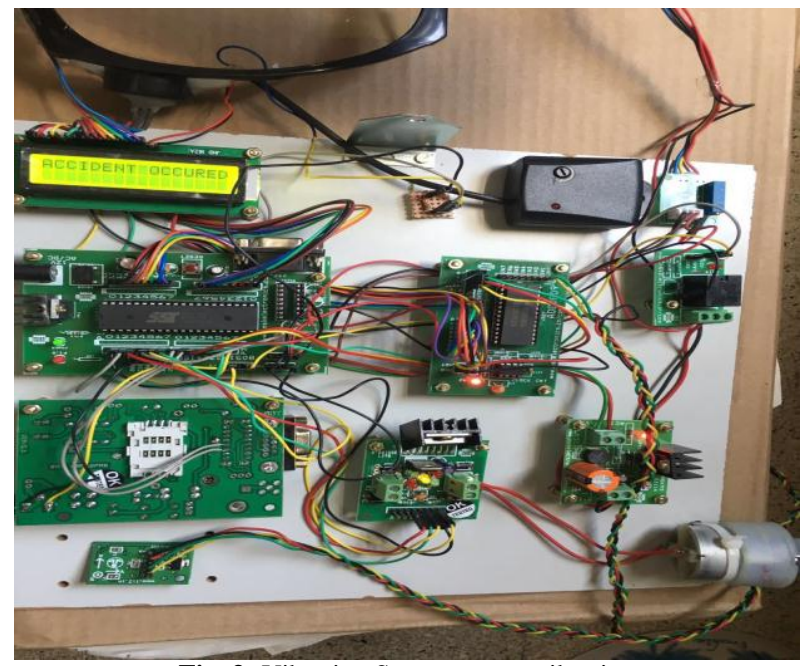

Fig. 3: Vibration Sensor senses vibration

Figure 3 shows that when a jerk or vibration is given to the sensor, the vibration sensor is activated and the wheel is stopped. The LCD displays a warning message as "ACCIDENT OCCURRED" and the vibrator on the eye blink sensor frame vibrates continuously. 


\subsection{Eye is shut}

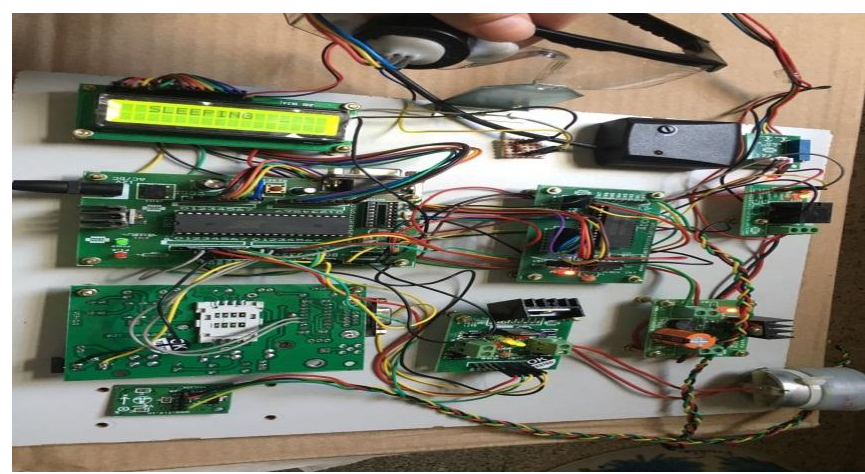

Fig. 4: Eye is shut

Figure 4 shows that when the eyes are closed then the vibrator vibrates and the speed of the wheel is decreased. The LCD displays a "SLEEPING" message.

\subsection{Location from Clamped Phone to Owner's Phone}

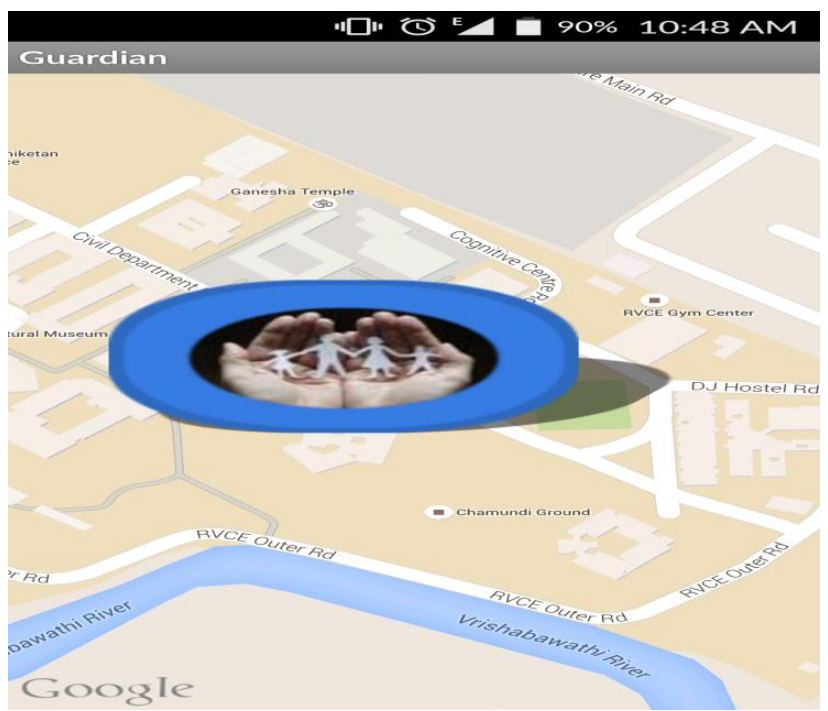

Fig. 5: Location from clamped phone to owner's phone

Figure 5 shows that location of the driver on the owner's phone. The owner first receives a text message containing latitude and longitude and then the location is displayed on google maps.

\subsection{Location Entered to Retrieve Nearby Police Station}

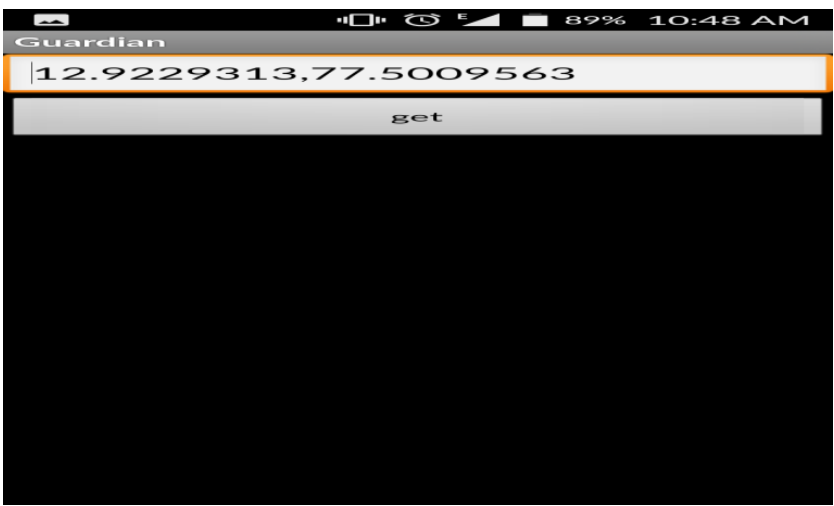

Fig. 6: Location entered to retrieve nearby Police Station

Figure 6 shows that after receiving the latitude and longitude from the clamped phone, those values are typed in the field shown in the figure. Then the option is selected to get the list of police stations

\subsection{List of Nearby Police Station}

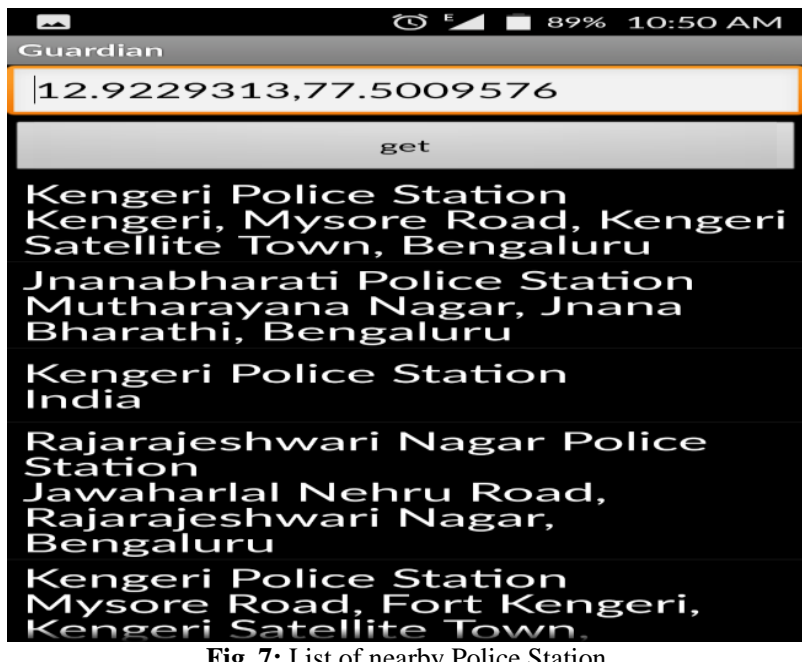

Figure 7 shows the list of nearby police stations to the accident location of the driver which is sent to owner's phone.

\subsection{Clamped Phone's Activity Screen}

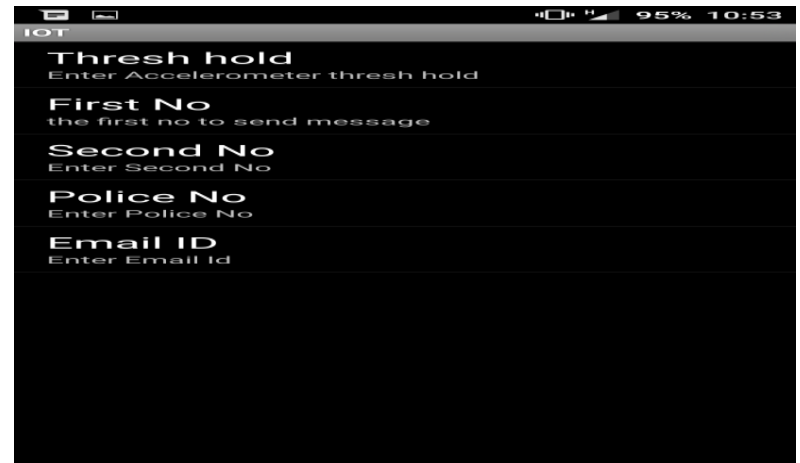

Fig. 8: Clamped phone's activity screen

Figure 8 shows the activity screen of clamped phone where the details of the owner are to be entered. The email address is entered here so that the photograph could be sent to the owner's email-id.

\subsection{Photograph Sent to Owner's Email-Id}

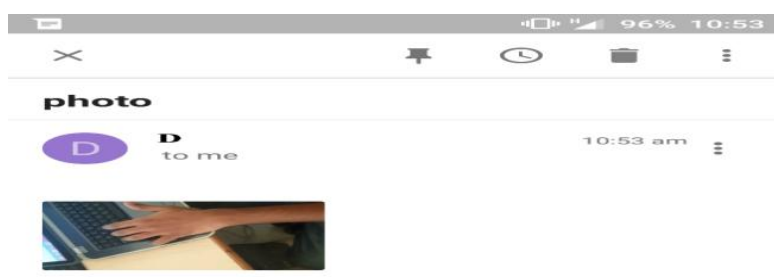

Fig. 9: Photograph sent to owner's email-id

Figure 9 Depicts the photograph received in the email of the owner when the owner chooses the photograph option.

\section{Conclusion}

The proposed system helpful to avoid vehicle accidents because of driver's sleepiness using eye blink sensor, in this paper we study 
and design the system for driver fatigue detection. If the driver becomes drowsy the eye blink sensor's frame vibrates attached to the vehicle and also the LCD displays the warning messages and it alerts the driver's through alarm sound to avoid the road accidents. The wheel is slowed or stopped depending on the condition. This is accompanied by the owner being notified through the GSM module, so the owner can retrieve the driver's location, photograph and a list of nearby police stations.

\section{References}

[1] MarcoJavier Flores, JoséMaría Armingol and Arturo de la Escalera, "Driver Drowsiness Warning System Using Visual Information for Both Diurnal and Nocturnal Illumination Conditions", Springer, EURASIP Journal on Advances in Signal Processing, 2010.

[2] Belhassen AkroutWalid Mahdi, "A Blinking Measurement Method for Driver Drowsiness Detection", Springer, Proceedings of the 8th International Conference on Computer Recognition Systems CORES, pp 651-660, 2013.

[3] Ji Hyun Yang, Zhi-Hong Mao, Member, IEEE, Louis Tijerina, Tom Pilutti, Joseph F. Coughlin, and Eric Feron, "Detection of Driver Fatigue Caused by Sleep Deprivation", IEEE TRANSACTIONS ON SYSTEMS, MAN, AND CYBERNETICS-PART A: SYSTEMS AND HUMANS, VOL. 39, NO. 4, JULY 2009.

[4] Antoine Picot, Sylvie Charbonnier, Alice Caplier, "On-Line Detection of Drowsiness Using Brain and Visual Information", Published in: IEEE Transactions on Systems, Man, and Cybernetics - Part A: Systems and Humans (Volume: 42, Issue: 3 ) Page(s): 764 - 775, May 2012.

[5] Satori Hachisuka, Kenji Ishida, Takeshi Enya, Masayoshi Kamijo, "Facial Expression Measurement for Detecting Driver Drowsiness", springer, International Conference on Engineering Psychology and Cognitive Ergonomics. EPCE 2011: Engineering Psychology and Cognitive Ergonomics pp 135-144, 2011.

[6] Ines Teyeb, Olfa Jemai, Mourad Zaied, Chokri Ben Amar, "A Drowsy Driver Detection System Based on a New Method of Head Posture Estimation", springer. International Conference on Intelligent Data Engineering and Automated Learning. IDEAL 2014: Intelligent Data Engineering and Automated Learning IDEAL 2014 pp 362-369, 2014.

[7] Nawal Alioua, Aouatif Amine, Mohammed Rziza, Driss Aboutajdine, "Driver's Fatigue and Drowsiness Detection to Reduce Traffic Accidents on Road", springer. International Conference on Computer Analysis of Images and Patterns, CAIP Computer Analysis of Images and Patterns pp 397-404, 2011.

[8] Esra Vural, Mujdat Cetin, Aytul Ercil, Gwen Littlewort, Marian Bartlett, Javier Movellan, "Drowsy Driver Detection Through Facial Movement Analysis", springer, International Workshop on Human-Computer Interaction HCI 2007: HumanComputer Interaction pp 6-18, 2007.

[9] Lin, Chin-Teng, et al. "Drowsiness estimation for safety driving using independent component analysis", Circuits and Systems I: Regular Papers, IEEE Transactions on 52.12: 2726-2738 pg-20-30, 2005.

[10] Clarke Sr, James Russell, and Phyllis Maurer Clarke, "Sleep detection and driver alert apparatus", U.S. Patent No. 5, 689, 241, pg25-70 18 Nov. 1997.

[11] Hayami, Takchito, et al. "Detecting drowsiness while driving by measuring eye movement-a pilot study", Intelligent Transportation Systems, Proceedings. The IEEE 5th International Conference on. IEEE, 2002 pg30-35, 2002

[12] Hu, Shuyan, and Gangtie Zheng, "Driver drowsiness detection with eyelid related parameters by Support Vector Machine", Expert Systems with Applications 36.4, pg651-658, 2009.

[13] Ito, Takehiro, et al, "Driver blink measurement by the motion picture processing and its application to drowsiness detection", Intelligent Transportation Systems, Proceedings. The IEEE 5th International Conference on. IEEE, 2002 pg30-35, 2002.

[14] Smith, Paul, Mubarak Shah, and Niels Da Vitoria Lobo, "Determining driver visual attention with one camera", Intelligent Transportation Systems, IEEE Transactions on 4.4: pg205-218, 2003.

[15] Kithil, Philip W., Roger D. Jones, and M. Jone, "Development of driver alertness detection system using overhead capacitive sensor array", SAE Technical Paper Series 982292: pg35-56, 1998

[16] Hong, Tianyi, Huabiao Qin, and Qianshu Sun, "An improved real time eye state identification system in driver drowsiness detection",
Control and Automation, 2007. ICCA 2007. IEEE International Conference on. IEEE, pg45-60, 2007.

[17] Ogawa, Kenji, and Mitsuo Shimotani, "A drowsiness detection system”, Mitsubishi Electric Advance: pg13-16, 1997.

[18] Yeo, Jung-hack. "Driver's drowsiness detection method of drowsy driving warning system", U.S. Patent No. 6,243,015. Pg:55-70, 5 June 2001.

[19] Flores, Marco Javier, José María Armingol, and Adl Escalera, "Real-time drowsiness detection system for an intelligent vehicle", Intelligent Vehicles Symposium, 2008 IEEE. IEEE, pg50-60, 2008.

[20] Wierwille, Walter W., et al, "Research on vehicle-based driver status/performance monitoring; development, validation, and refinement of algorithms for detection of driver drowsiness", Final report. No. HS-808 247, pg-30-33, 1994.

[21] Yang, Ji Hyun, et al, "Detection of driver fatigue caused by sleep deprivation", Systems, Man and Cybernetics, Part A: Systems and Humans, IEEE Transactions on 39.4: pg694-705, 2009.

[22] Bergasa, Luis M., et al, "Real-time system for monitoring driver vigilance",Intelligent Transportation Systems, IEEE Transactions on 7.1: pg.63-77, 2006. 